Journal of Engineering and Applied Sciences 15 (2): 523-528, 2020

ISSN: 1816-949X

(C) Medwell Journals, 2020

\title{
Automated pH Monitoring and Controlling System for Hydroponics under Greenhouse Condition
}

\author{
Amy Lizbeth J. Rico \\ College of Engineering and Technology, Malacampa, Camiling, \\ 2306 Tarlac, Philippines
}

\begin{abstract}
An automated $\mathrm{pH}$ monitoring and controlling system for hydroponic system was developed, calibrated and validated in this study. The automated monitoring and controlling system was developed to monitor and control the $\mathrm{pH}$ of nutrient solution in hydroponic system. The greenhouse available at the Center for Hydroponics and Aquaponics Technology (CHAT) and locally available materials and hardware for the hydroponics and automation were used in the development of the system. These devices were designed and assembled based on the conceptual framework of the study. The $\mathrm{pH}$ sensor sends signal to the microcontroller which triggers the adding of acid/base solution and turning on/off of the mixer. The instruments used were calibrated prior to the performance evaluation and obtained calibration equation for the $\mathrm{pH}$ sensor is $\mathrm{y}=0.91 \mathrm{x}+0.91$. Validation of the automated $\mathrm{pH}$ monitoring and controlling system was done and the recorded maximum $\mathrm{pH}$ is 6.54 and minimum $\mathrm{pH}$ of 6.0. The lettuce planted during the validation has an average height of $14.61 \mathrm{~cm}$ and the average leaf count of 12 for the lettuce crops during the 4th week after planting. A total of $4.78 \mathrm{~kg}$ of lettuce crop was harvested with an average of $20.6 \mathrm{~g}$ per lettuce crop was obtained. Based on the performance evaluation and validation done on the automated $\mathrm{pH}$ monitoring and controlling system, it was found to be reliable. This system becomes useful in reducing labor cost and allows for real-time monitoring of $\mathrm{pH}$, therefore, increasing farmer's crop productivity and income.
\end{abstract}

Key words: Validation, framework, evaluation, calibration, $\mathrm{pH}$, hydroponics

\section{INTRODUCTION}

Agriculture plays an important part in the growth and development of a country. Having an agricultural industry allows a country to produce its own food which does not necessitate the need for the trade and importation of goods from other countries. In short, agriculture allows for the self-sufficiency of a country in terms of its food supply and other agricultural products (Tagle et al., 2018). Hydroponics system is a growing plant system that does not use soil but uses water with nutrients to save space planting and not contaminated with chemicals in the soil (Kularbphettong et al., 2019). The only requirements with the water culture system is a water reservoir to supply nutrients to the plants, a platform for the plants on top of the nutrient solution. Water culture systems are the systems of leafy lettuce. These are very inexpensive systems which can be utilized in areas with little to no electricity. In the present scenario, almost everything can be controlled and operated automatically, systems become smarter and automatic in that way there is a need of revolution in agriculture industry (Mishra and Jain, 2015). Arduino uno microcontroller can be used to implement nutritional water flow system and the sensors can well detect and handle the water level in hydroponic tubes
(Sihombing et al., 2018). Though, there are several other parameters such as air temperature and humidity, lights, water temperature, etc. which are not controlled or maintained. These parameters are important for a healthy and faster plant growth (Brechner et al., 2013).

Hence, this study is conceptualized to develop an automated system by monitoring the $\mathrm{pH}$ of the nutrient solution in a hydroponic system for optimum plant growth as this factor can greatly affect the growth of lettuce. Specifically, the study aimed to install an automated $\mathrm{pH}$ monitoring and controlling mechanism for the nutrient solution, evaluate the performance of the automated monitoring and controlling device and determine the response of lettuce on the automated monitoring and controlling device.

\section{MATERIALS AND METHODS}

Conceptualization of the study: The conceptual paradigm of the study is presented in Fig. 1. The study aimed to monitor and control the $\mathrm{pH}$ of the nutrient solution using hydroponic system under greenhouse condition. Through this process, time and labor can be saved as well as real time monitoring of the parameters can be achieved. 
J. Eng. Applied Sci., 15 (2): 523-528, 2020

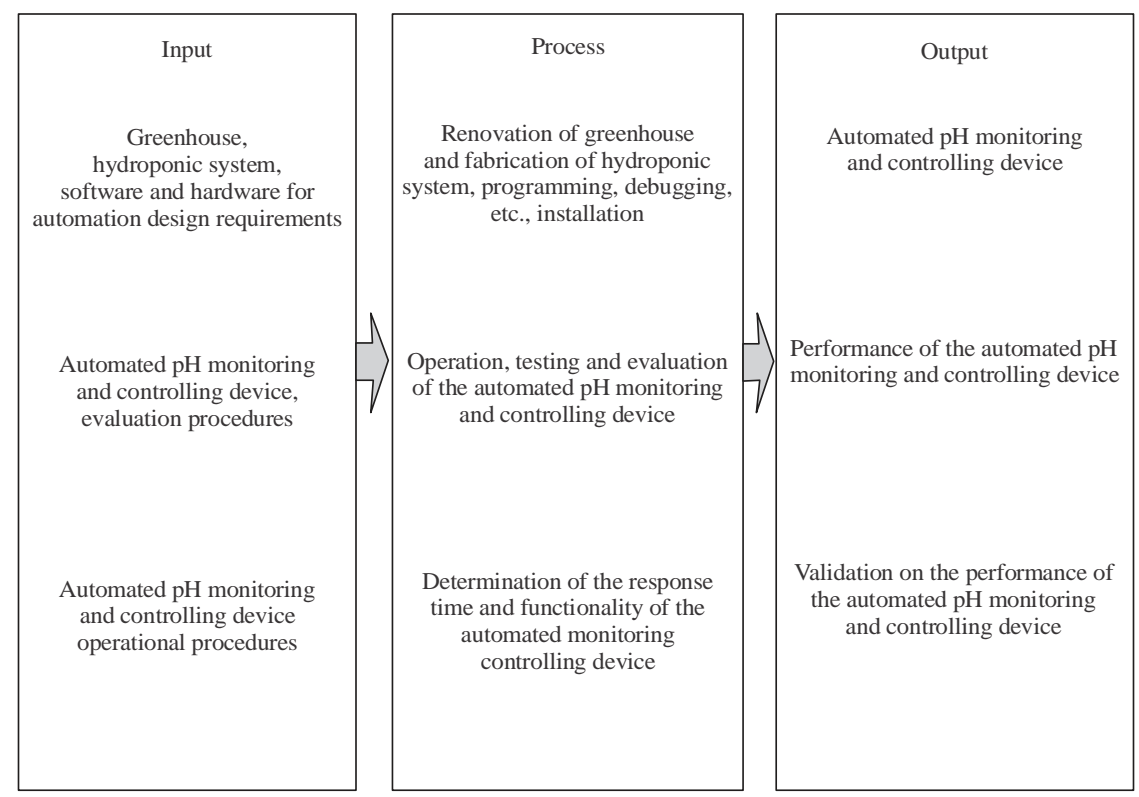

Fig. 1: Conceptual framework of the study

The production system: The automated hydroponic system used in the study is composed of the structural system, the hydroponic system and the automation system.

The automated $\mathrm{pH}$ monitoring and controlling was tested in one of the greenhouse facilities located at the Center for Hydroponics and Aquaponics Technology (CHAT) measuring $3.0 \mathrm{~m}$ in width, $4.0 \mathrm{~m}$ in length and $3.5 \mathrm{~m}$ in height. The frames of the greenhouse are made from $2.54 \mathrm{~cm}$ galvanized iron pipes bended and welded together to form a quonset-type structure. The structure is provided with three roof covers: the insect-proof net in the inner side, the ultraviolet-resistant plastic film in the middle and the gravy woven net shade on the outer side that offers strength and improve the ventilation in the system.

The recirculating tube culture system was used in hydroponic system. The hydroponic system was enclosed in the structural system. The grow pipes used was $300.0 \mathrm{~cm}$ in length and $0.075 \mathrm{~cm}$ diameter. A slope of $1 \mathrm{~cm} / 100 \mathrm{~cm}$ of the pipe length was employed for the water to flow through the pipe with ease. The PVC pipes were drilled with $5.08 \mathrm{~cm}$ diameter holes and were spaced at $16.5 \mathrm{~cm}$ between holes (center to center) and made in 2-layer and 4-column pipe layout. A $150 \mathrm{~L}$ reservoir served as the source of water in the hydroponics system where the water was pumped to each growing tubes. The water flow in the hydroponic system was run by a $65 \mathrm{~W}$ submersible pump, 1-2 $\mathrm{L}^{-1} \mathrm{~min}$ flow for each growing tube that lifts the water to the upper layer of the growing tubes. A mixer inside the reservoir was installed to equally dispense the nutrient solution to the reservoir water.
Figure 2 shows the set-up of the automated $\mathrm{pH}$ monitoring and controlling device. The automation system served as the main component of the study and was composed of the controls, sensors and hardware.

Automation of the $\mathrm{pH}$ monitoring and controlling device: The automated $\mathrm{pH}$ monitoring and controlling device basically monitor and control the parameters such as $\mathrm{pH}$ of the nutrient solution in a hydroponic system under greenhouse condition. Sensors were used to determine the $\mathrm{pH}$ in the reservoir. The block diagram shown in Fig. 3 is the layout of the hardware design that was used for the automated monitoring and controlling device. A microcontroller using the Arduino platform was used in programming the automation of the $\mathrm{pH}$ monitoring and controlling device. Using this data, the microcontroller adjusts the $\mathrm{pH}$ level of the water in the system by adding acid and base solution and turning the mixer on.

Figure 4 is the flow diagram of the automation used in the study. The LCD is initialized when the automation system is turned on. The $\mathrm{pH}$ range of $6.0-6.8$ for the nutrient solution was entered in the system. These ranges determine when the acid/base solutions will be added and the mixer will be turned on and determined using the $\mathrm{pH}$ level sensor submersed into the reservoir. If the $\mathrm{pH}$ level reading is below 6.0, the sensor sends signal to the microcontroller to trigger the peristaltic pumps to add base solution as well as to turn on the mixer. On the other hand if the $\mathrm{pH}$ level of the nutrient solution is above 6.8, 


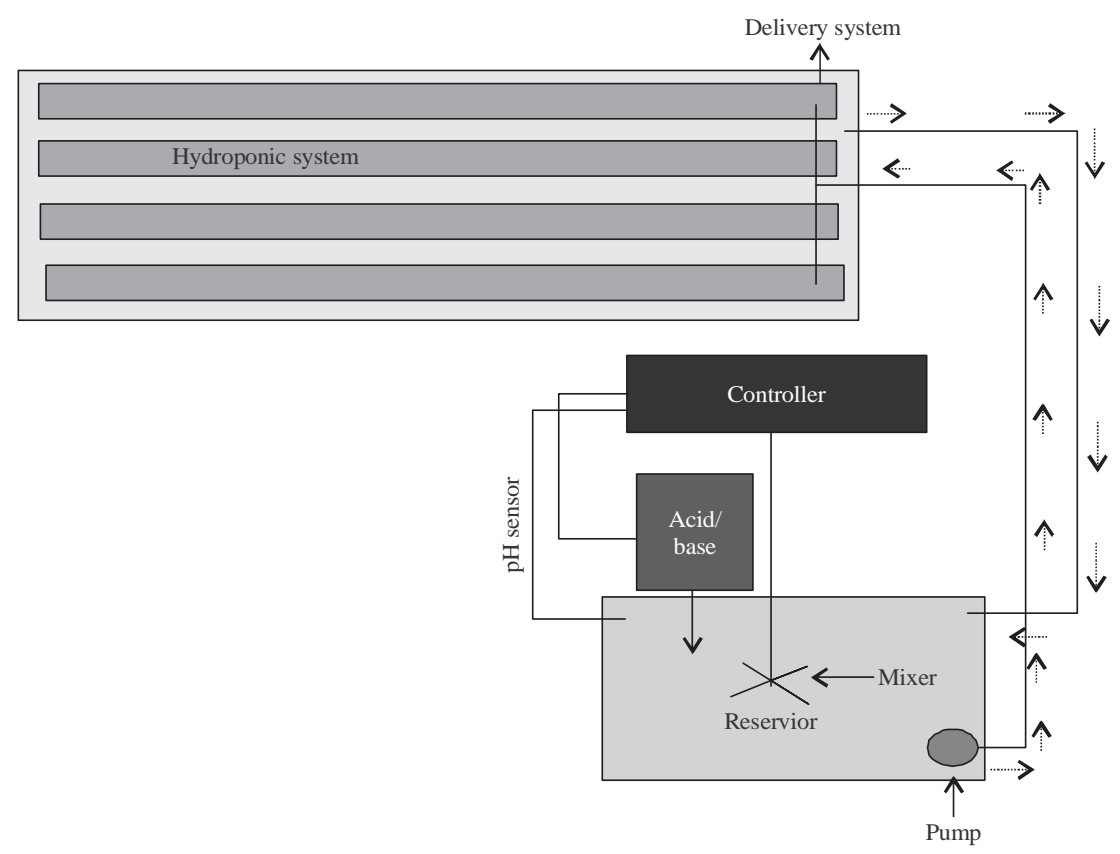

Fig. 2: Set-up of the automated $\mathrm{pH}$ and temperature monitoring and controlling device

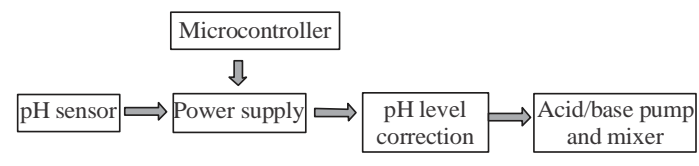

Fig. 3: Block diagram of the automated $\mathrm{pH}$ and temperature monitoring and controlling system

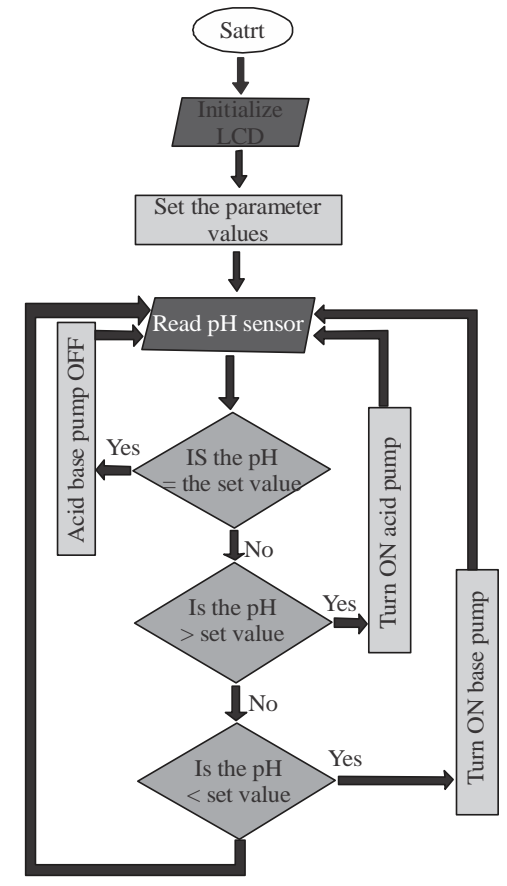

Fig. 4: Flow diagram of the automated system the sensor sends signal to the microcontroller which triggers the peristaltic pumps to add acid solution and turn on the mixer. When the entered $\mathrm{pH}$ range is attained, the sensors send signal the microcontroller to turn off the mixer and the addition of base/acid solution in the reservoir.

Calibration of the $\mathbf{p H}$ sensor: The $\mathrm{pH}$ sensor was calibrated in order to achieve precision and accuracy. The hourly reading for $24 \mathrm{~h}$ period in the sensor was compared with the reading from the calibrated instruments. The difference in reading from the sensor and the calibrated instrument were recorded and graphed. Linear regression of the sensor reading and the calibrated instruments was obtained. The equation from the linear regression was inputted into the program for the $\mathrm{pH}$ sensor.

Final testing: The $\mathrm{pH}$ was monitored every day based on their response to the whole system. Automatic turning on of the device when the parameters are beyond the threshold range, response time of the device to be able to attain the threshold range and automatic turning off of the devices when threshold range is attained were among the data gathered and recorded.

Lettuce production: The leafy variety of lettuce (Lollo rossa) was used as planting material in the automated hydroponics system as this is commonly used as planting material in hydroponics system. Media composed of 
carbonized rice hull, sand and rice hull was used as planting media in the automated hydroponics system, since, these contain most nutrients needed by the plants. The planting cups containing 2-3 lettuce seeds were placed in cups. The cups were placed on individual cut-outs of the growing tubes. The net cups should touch the flowing water in the growing tubes to avoid the plants to be dehydrated. The pump continuously lifts the water and nutrient solution allowing the roots to avail of the nutrients. The $\mathrm{pH}$ level of the nutrient solution was maintained at a range of 6.0-6.8 level which is the recommended $\mathrm{pH}$ level for lettuce production under hydroponics system. At this $\mathrm{pH}$ level, the needed nutrients were made available to the lettuce plants. These parameters were maintained throughout the growing stage until harvesting stage of the lettuce. The lettuce was harvested 27 days after planting.

Validation: Validation refers to the process of checking that a system meets the specifications and that it fulfils its intended purpose. In the automated hydroponics system, the data gathered from the final testing was analysed and graphed. The automation system was modified to optimize the production system based on the data gathered.

Another growing cycle of the lettuce was planted in the automated hydroponics system. Response of the system was monitored from planting to harvesting of the lettuce. The gathered data during validation was compared from the gathered data from the final testing. The differences from the two growing cycle and their relationship was obtained.

\section{RESULTS AND DISCUSSION}

The automated $\mathrm{pH}$ monitoring and controlling system for hydroponics under greenhouse condition was calibrated, monitored and evaluated to determine its performance. Daily monitoring of the $\mathrm{pH}$ and temperature of the nutrient solution was recorded and graphed for 27 days and its performance was evaluated. The height, number of leaves and yield of the lettuce were recorded and graphed to validate the response of the lettuce crops in the automated system.

Installation of the automated $\mathrm{pH}$ monitoring and controlling system for nutrient solution: The microcontroller used in the automated hydroponics system is Arduino Mega 2560 which served as the brain of the system and served as the trigger. It also processes the sensor data. Most of the parts were connected to the Arduino using simple jumper wires and the wires were soldered to ensure that they would not get loose. All of the electronic parts were then placed into plastic enclosure (Fig. 5 and 6) to protect delicate electronic parts from dust and moisture.

\section{Monitoring device}

pH sensor: One of the important requirements of the automated hydroponic system is the measurement and adjustment of the $\mathrm{pH}$ of the hydroponics reservoir. In order to know when the $\mathrm{pH}$ chemical needs to be added to the hydroponics reservoir, a pH sensor (Fig. 7) reads the $\mathrm{pH}$ continuously. The $\mathrm{pH}$ sensor was used for determining the acidity level of the reservoir. It sends trigger signal to the microcontroller to activate the peristaltic pumps for the acid/base solution and the mixer. The peristaltic pumps dispense $1.0 \mathrm{~mL} / \mathrm{sec}$ of acid/base when activated and stops when the threshold range is attained.

Peristaltic pump: One of the important components of the automated $\mathrm{pH}$ controlling system is the peristaltic pump. The peristaltic pumps were connected to the Arduino board. The $\mathrm{pH}$ sensor sends signal to the microcontroller whenever the $\mathrm{pH}$ reading is beyond the threshold limit and triggers the peristaltic pump to add acid or base to the reservoir. Figure 8 shows the peristaltic pumps for the addition of acid and base in the automated system.

Calibration of the $\mathrm{pH}$ monitoring and controlling system: Calibration of the $\mathrm{pH}$ sensor sensor used was done at the Center for Hydroponics and Aquaponics Technology (CHAT) in a $24 \mathrm{~h}$ period before the data gathering. The reading from the sensors and calibrated instruments was obtained, recorded and graphed. The graph of the calibration for $\mathrm{pH}$ sensor is shown in Fig. 9 and the obtained calibration equation is $\mathrm{y}=0.91 \mathrm{x}+0.9$. This equation was inputted in the program for the automation of the hydroponics system.

Performance evaluation: Based on the results, the obtained maximum $\mathrm{pH}$ is 6.82 and minimum $\mathrm{pH}$ is 6.0. The ability of the system to respond to the set threshold level, the response time of the system to the parameters and the difference from the calibrated instrument were observed to be able to determine the reliability of the automated hydroponics system. Results showed that turning on of the peristaltic pumps for acid and base and turning on of the mixer and chiller thermostat when the reading is beyond the threshold range is attained immediately after the reading is beyond the set value in the hydroponics system.

Validation of the automated $\mathrm{pH}$ monitoring and controlling system: During the validation period, the 


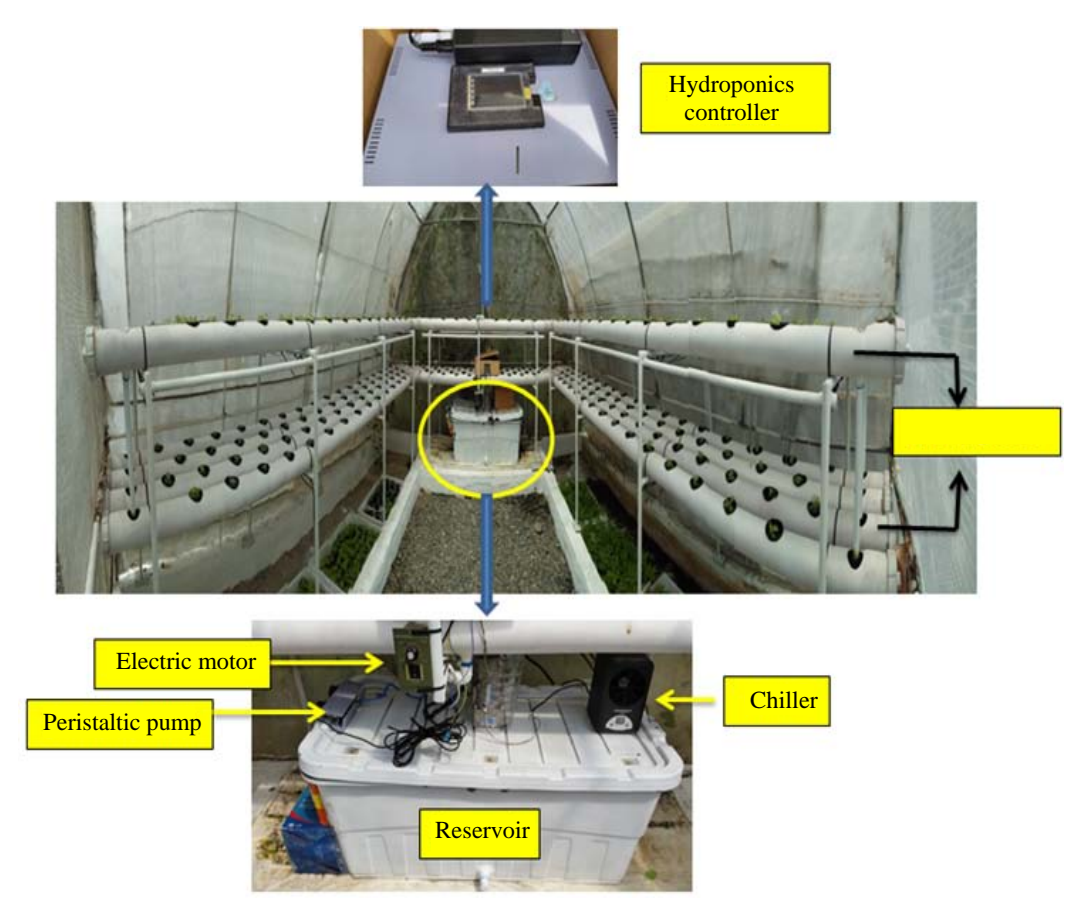

Fig. 5: The hydroponic system

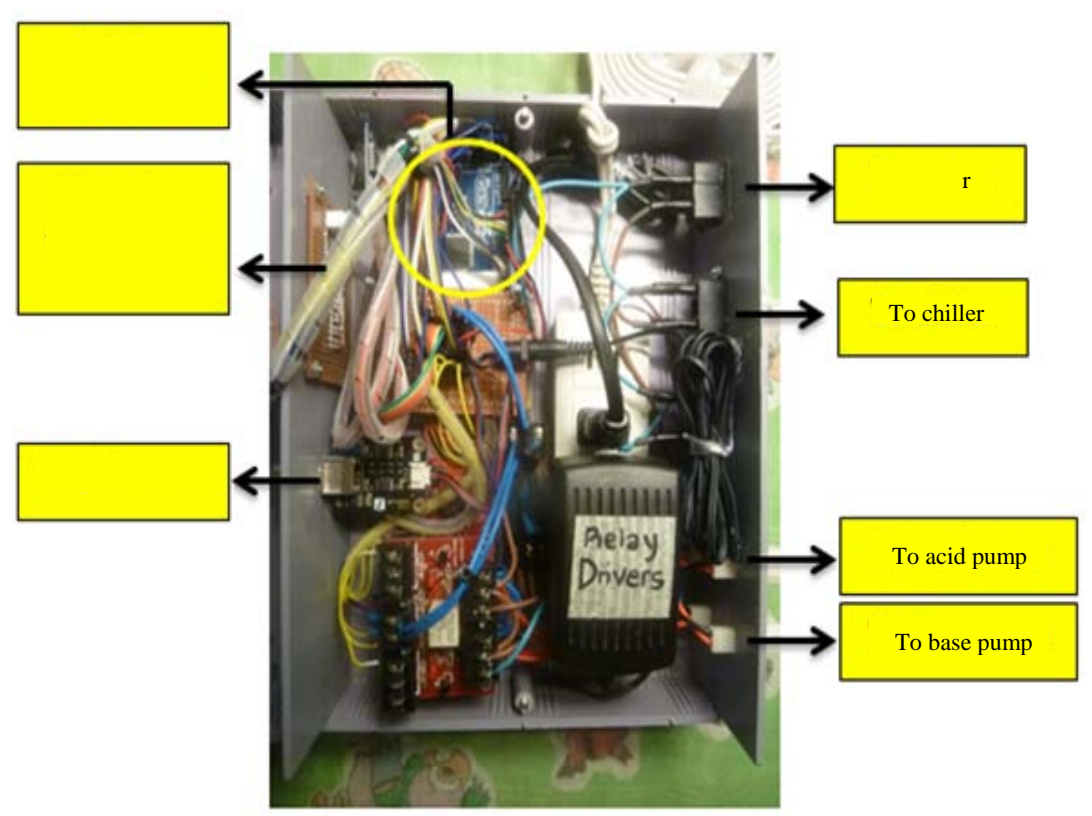

Fig. 6: Electronic parts used in the automated hydroponic system

system was observed based on the criteria set for the $\mathrm{pH}$. Based on the results, the $\mathrm{pH}$ reading and responses were accepted during the validation. Similar performance of the system during the validation and during the performance evaluation was observed. During the validation of the automated $\mathrm{pH}$ and temperature monitoring and controlling system, the growth and number of leaves of the lettuce (test crop) were gathered and recorded weekly and the yield of the lettuce was obtained during harvesting. The lettuce crops obtained a total yield of $4.78 \mathrm{~kg}$ and an average of $20.6 \mathrm{~g}$ per crop (Fig. 7-9). 


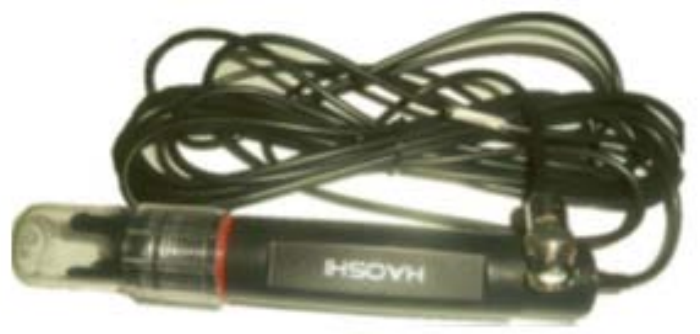

Fig. 7: $\mathrm{pH}$ sensor used in the automated hydroponic system

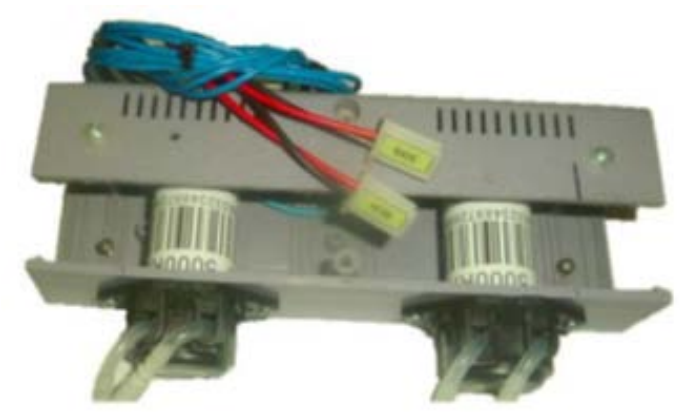

Fig. 8: The peristaltic pump that pumps acid and base to the reservoir

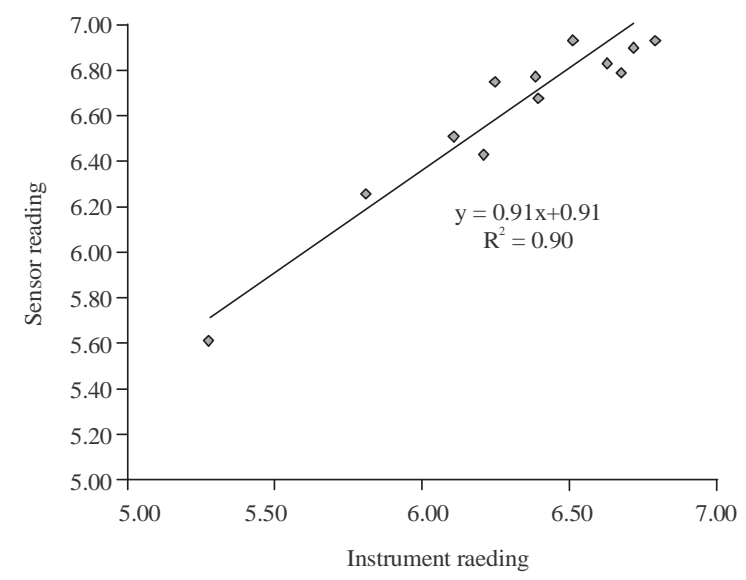

Fig. 9: Calibration curve for $\mathrm{pH}$ sensor

\section{CONCLUSION}

Based on the objectives, the following conclusions were drawn: the installed automated $\mathrm{pH}$ controller was able to maintain the desired condition for the hydroponic system based on the observed successes and failures in monitoring the $\mathrm{pH}$, the performance of the developed automated $\mathrm{pH}$ controller was found to be reliable and the automated $\mathrm{pH}$ controlling and monitoring device was able to grow lettuce with yield and responses similar to normal growing conditions.

\section{ACKNOWLEDGEMENT}

This humble piece of research would not have been successful without the big heart of wonderful people who have given their endless support, motivation and guidance and to whom I am profoundly indebted. I would like to acknowledge and express my sincerest gratitude to the following:

To the Department of Science and TechnologyEngineering Research and Development for Technology (DOST-ERDT) and the College of Engineering-Central Luzon State University, for all the support accorded to me. Sincere appreciation is given to Dr. Ireneo C. Agulto, the hardworking Dean of the College of Engineering and Project Leader of DOST-ERDT for accommodating and providing the most needed research grant. Sincere gratitude is also extended to Dr. Emmanuel V. Sicat for the scholarly advice, intelligent suggestions and persuasive motivation he had given me during the entire course of this research.

\section{REFERENCES}

Brechner, M., A.J. Both and C.E.A. Staff, 2013. Hydroponic lettuce handbook. Cornell University, Ithaca, New York, USA. http://cea.cals.cornell.edu/attachments/Cornell\%20 CEA\%20Lettuce\% 20Handbook\%20.pdf

Kularbphettong, K., U. Ampant and N. Kongrodj, 2019. An automated hydroponics system based on mobile application. Int. J. Inf. Educ. Technol., 9: 548-552.

Mishra, R.L. and P. Jain, 2015. Design and implementation of automatic hydroponics system using ARM processor. Int. J. Adv. Res. Electr. Electron. Instrum. Eng., 4: 6935-6940.

Sihombing, P., N.A. Karina, J.T. Tarigan and M.I. Syarif, 2018. Automated hydroponics nutrition plants systems using Arduino UNO Microcontroller based on android. J. Phys. Conf. Ser., Vol. 978,

Tagle, S., H. Benoza, R. Pena and A. Oblea, 2018. Development of an indoor hydroponic tower for Urban farming. Proceedings of the 6th DLSU International Conference on Innovation and Technology Fair, November 22-23, 2018, De La Salle University, Manila, Philippines, pp: 1-7. 